\title{
Lithium abundances of nearby young solar-type stars based on optical high-resolution spectroscopy
}

\author{
L.-F. Xing ${ }^{1}$ and Q.-F. Xing ${ }^{2}$ \\ 1 Department of Technology and Physics, Zhengzhou University of Light Industry, 5 Dongfeng Road, 45002 Zhengzhou, PR China \\ e-mail: lfxing@bao.ac.cn \\ 2 National Astronomical Observatories, Chinese Academy of Sciences, 100012 Beijing, PR China
}

Received 25 April 2011 / Accepted 4 November 2011

\begin{abstract}
Context. Lithium has long been recognized as a powerful tool for investigating the internal mixing of low-mass stars. In addition, accurate measurements of lithium abundances in young solar-type stars provide independent and reliable age diagnostics.

Aims. To study the relationship between lithium and activity in normal solar type-type stars and determine the effectiveness of lithium and kinematics as activity indicators, we perform a lithium survey of nearby late-type stars. We search for nearby young solar-type stars and investigate the relationship between lithium abundances and X-ray activity by measuring $\log L_{\mathrm{x}}$.

Methods. On the basis of high-resolution spectroscopic observations, we derived the lithium abundances of 33 young solar-type stars by comparing the measured $\mathrm{Li}$ I $\lambda 670.8 \mathrm{~nm}$ equivalent widths with the curve of growth calculations in non-LTE (NLTE) conditions. Results. We obtain the lithium abundances of a sample of young solar-type stars. Using the lithium abundance and X-ray luminosity, $L_{\mathrm{x}}$, we investigate the relationship between Li abundance and X-ray activity. We find a clear correlation between lithium abundance $(\log N(\mathrm{Li}))$ and $\mathrm{X}$-ray luminosity, $\log L_{\mathrm{x}}$, for our sample stars.

Conclusions. As the X-ray luminosity, $L_{\mathrm{x}}$, becomes stronger, the lithium abundance decreases in our sample (11 pre-main-sequence, 15 zero-age-main-sequence, and 7 young main-sequence) stars. The results imply that there is a large number of solar-type stars. A correlation appears to exist between $\mathrm{Li}$ abundance and age, thus confirming the presence of very active young stars close to the Sun.
\end{abstract}

Key words. stars: evolution - stars: abundances - stars: solar-type

\section{Introduction}

Solar-type stars are low-mass, late spectral-type stars. They are similar to the Sun in mass and evolutionary state. Physically, they have a broadly similar structure and a convective envelope; however, they do not have completely dominant convection, as found in M dwarfs. Young solar-type stars (a weak-line T Tauri star (WTTS), and low-mass zero-age-main-sequence (ZAMS), and young main-sequence (MS) stars) display various forms of activity caused by dynamic processes.

Lithium has long been recognized as a powerful tool for investigating the internal mixing of low-mass stars. Since lithium isotopes in low-mass stars are destroyed by proton capture at low temperatures, they allow us to directly probe the depths of outermixed envelopes. Observations of field stars and open clusters clearly show that the destruction rate of Li depends on the mass, age, and chemical composition of a star (Duncan 1981; Spite \& Spite 1982; Duncan \& Jones 1983; Cayrel et al. 1984; Boesgaard \& Tripicco 1986; Hobbs \& Pilachowski 1986; Rebolo et al. 1988). In particular, different authors have proposed empirical expressions for Li abundance as a function of star age (Rebolo 1989; Boesgaard 1991).

Increasing evidence has indicated that dispersion in $\mathrm{Li}$ abundances exists among stars with similar ages, metallicities, and masses (Soderblom et al. 1993a; Boesgaard et al. 1998). This dispersion suggests that some other stellar property is important in determining the amount of Li depletion in stars. Observational data clearly indicate that rotation plays a key role in determining the amount of Li depletion in a star (Barrado y Navascués \& Stauffer 1996; Jones et al. 1997). In addition, there is clear evidence of a correlation between rotation rates and $\mathrm{Li}$ abundances in the Pleiades and Hyades, with the fastest rotators having the highest Li abundance (Tschäpe \& Rüdiger 2001; Rebolo \& Beckman 1988). In particular, the X-ray surface flux is a good measure of stellar activity because it shows a clear dependence on stellar rotational velocity (Preibisch 1997). The enhanced X-ray emission displayed by rapid rotators (T Tauri stars and RS CVn systems) relative to main-sequence stars can be accounted for by their higher rotational velocities (Bouvier \& Bertout 1985). Therefore, a correlation can be expected between lithium abundances and star activity.

To study the relationship between lithium and X-ray activity in normal solar-type stars, as well as determine the effectiveness of lithium and kinematics as activity indicators, we performed a survey of lithium over a sample of nearby dwarfs. The aim of the current paper is to investigate the relation of lithium abundances to X-ray activity in terms of $\log L_{\mathrm{X}}$. The observations and data reduction are presented in Sect. 2. The analysis of lithium abundance is provided in Sect. 3, and the results and discussion are presented in Sect. 4.

\section{Observations and data reduction}

Spectroscopic observations were carried out for thirteen nights from January 15 to 17,2008 , October 6 to 8, 2009, January 26 to 28, 2010, and February 18 to 21, 2011 with the Coude Echelle 


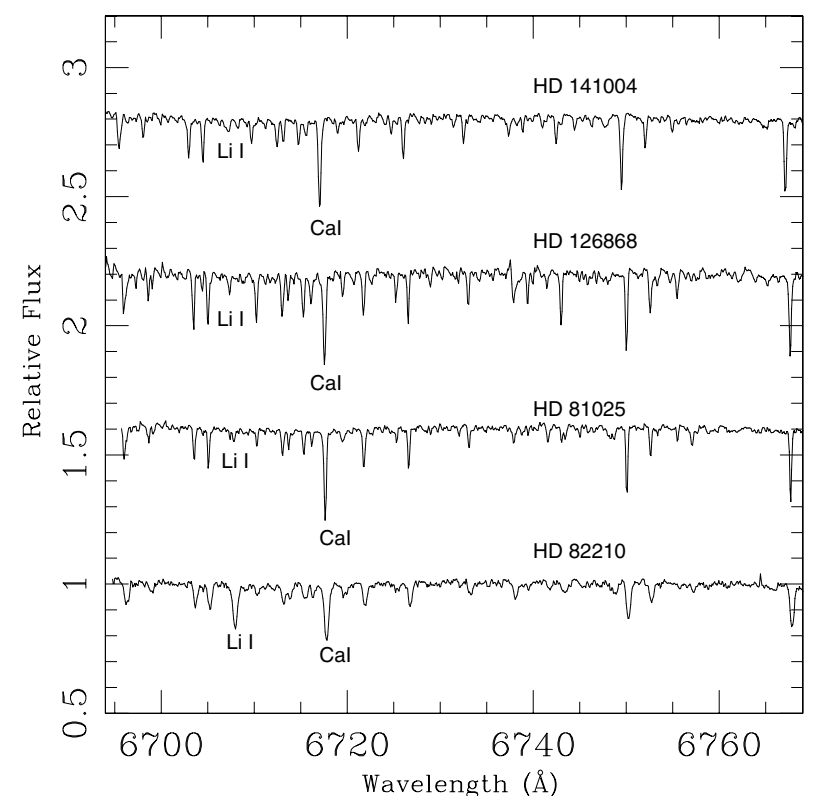

Fig. 1. Examples of the spectra of stars with a range of Li I $\lambda 6707 \mathrm{~m} \AA$ lines.

Spectrograph and a $1024 \times 1024$ Tek CCD attached to the $2.16 \mathrm{~m}$ telescope at the Xinglong station of the National Astronomical Observatories, Chinese Academy of Science (NAOC). The red arm of the spectrograph with a 31.6 groove $/ \mathrm{mm}$ grating was used in combination with a prism as the cross-disperser, which provided good separation between the echelle orders (Zhao \& Li 2001). With a $0.5 \mathrm{~mm}$ slit (1.1 arcsec), the resolving power was of approximately 37000 . The exposure time was chosen to ensure that the signal-to-noise ratio $(S / N)$ was higher than 100 , and the spectral coverage is $580-880 \mathrm{~nm}$. This relatively high resolution was judged to be important in view of the relatively weak lithium lines in some stars.

First, all observational data were reduced using the Image Reduction and Analysis Facility (IRAF) ${ }^{1}$ software package in the standard fashion, including image trimming, bias subtraction, flat-field division, spectrum extraction, and cosmic ray removal. The wavelength calibration was then obtained by taking the spectra of a Th-Ar lamp. Finally, all spectra were normalized using a cubic spline fit to the observed continuum. Figure 1 shows the spectra of four sample stars in the range of the $\mathrm{Li} \mathrm{I} \lambda$ $670.8 \mathrm{~nm}$ line.

To determine the $\mathrm{Li}$ I $\lambda 670.8 \mathrm{~nm}$ equivalent widths $(\mathrm{EW}(\mathrm{Li}))$, all spectral lines of the relevant order were averaged to find regions in the continuum unaffected by metal lines. These regions were then used to fit the continuum with a straight line. The EW (Li) was then determined by fitting a Gaussian curve. The nominal resolving power $\lambda / \Delta \lambda$ was $10^{5}$, which was sufficient to resolve the $\mathrm{Li}$ I feature from a nearby Fe I line at $6707.44 \AA$ in all regions. Since we observed bright stars and the seeing conditions were generally good, the $S / N$ of our observations was high, between 100 and 300 .

Following Palla et al. (2005), the relationship between the true and measured equivalent widths (EWs) is $\mathrm{EW}_{\text {true }}=$ $\mathrm{EW}_{\text {meas }}(1-r)$, where $r$ is the ratio of the excess to the photospheric continuum. To estimate $r$, we measured the EWs of

\footnotetext{
${ }^{1}$ IRAF is distributed by the National Optical Astronomy Observatories, which is operated by the Association of Universities for Research in Astronomy, Inc., under contract with the National Science Foundation.
}

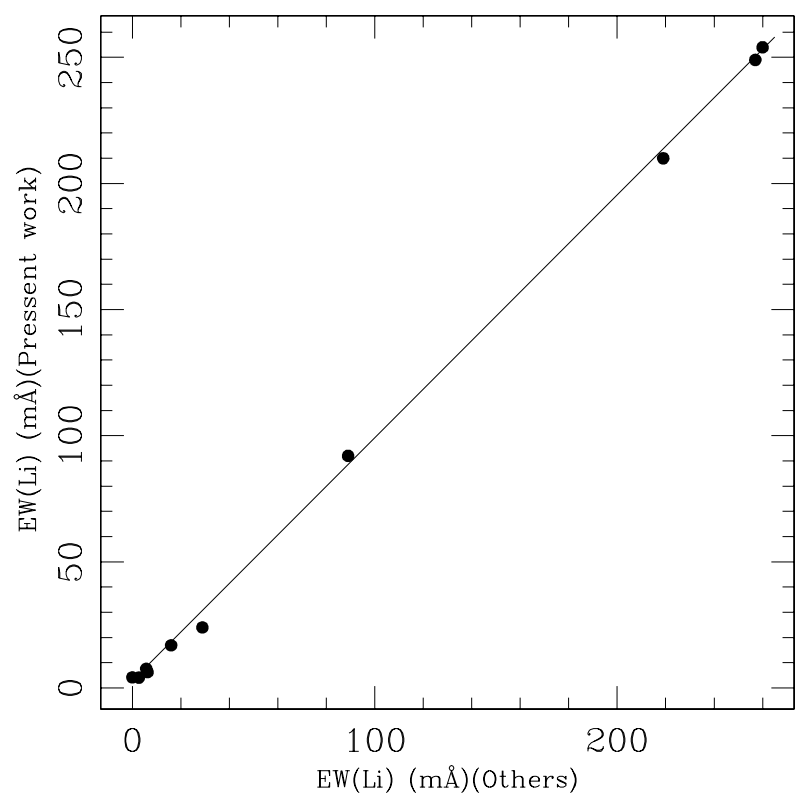

Fig. 2. Comparison of our measurement of the Li equivalent width obtained with a Gaussian fit and similar measurements obtained by other authors for the same stars (Christian \& Mathioudakis 2002; Wichmann et al. 2000; Gregorio-Hetem \& Hetem 2002; de Laverny et al. 2003; Takeda \& Kawanomoto 2005; Jasniewicz et al. 1999; Montes et al. 2001).

three strong lines included in our spectral range (Ni I $664.36 \mathrm{~nm}$, Fe I $666.34 \mathrm{~nm}$, and V I $662.48 \mathrm{~nm}$ ) for all target stars. We then compared them with those measured in the spectra of stars of similar temperature in IC 2391 and IC 2602, which were old enough (30-50 Myr, Randich et al. 2001) to ensure that their spectra are not affected by veiling. Therefore, we did not attempt any veiling correction in our young solar-type stars.

In Fig. 2, we compare our measurement of the Li EWs with those obtained by other authors for the same stars (Christian \& Mathioudakis 2002; Wichmann et al. 2000; Gregorio-Hetem \& Hetem 2002; de Laverny et al. 2003; Takeda \& Kawanomoto 2005; Jasniewicz et al. 1999; Montes et al. 2001). The agreement between the values was good; however, for small EWs, we noted some differences between the samples of other authors and our results. We found an agreement of closer than $15 \%$ for 8 stars when compared with the literature data, and we estimated an average error bar of $5 \%$ in the $\mathrm{EW}$ values at $1 \sigma$, resulting mainly from uncertainties in the continuum placement.

\section{Lithium abundances of young solar-type stars}

\subsection{Effective temperatures and surface gravity}

The effective temperature of our 33 sample stars was determined from the line-depth ratios of VI $\lambda 6251.83$ and Fe I $\lambda$ 6252.527, using the calibrations of Gray and Johanson (1991). The line depths were measured with a ruler on a plot of the profiles (Xing 2010). We also determined the effective temperature for 33 program stars from the $(B-V)$ color index, using the calibrations of Casagrande et al. (2006). The effective temperature of program stars derived from different methods is listed in Table 1.

The gravity is determined via Hipparcos parallaxes according to the method given by Nissen et al. (1997).

Following the uncertainty analysis of Chen et al. (2000), we can estimate the mean uncertainty in $\log g$. The largest uncertainty in the gravity comes from the parallax. A typical relative 
Table 1. Photometric results and other parameters of our sample of stars.

\begin{tabular}{|c|c|c|c|c|c|c|c|c|c|c|c|}
\hline$\#$ & Stars & SpT. & V & $B-V$ & $\begin{array}{l}T_{\text {eff }}^{C} \\
(\mathrm{~K})\end{array}$ & $\begin{array}{l}T_{\text {eff }}^{R} \\
(\mathrm{~K})\end{array}$ & $\begin{array}{c}\mathrm{EW}(\mathrm{Li}) \\
(\mathrm{m} \AA)\end{array}$ & $\log N(\mathrm{Li})$ & $\begin{array}{c}\mathrm{EW}\left(\mathrm{H}_{\alpha}\right) \\
(\mathrm{m} \AA)\end{array}$ & $\begin{array}{l}\log L_{\mathrm{x}} \\
(\mathrm{erg} / \mathrm{s})\end{array}$ & $\log F_{\mathrm{s}}$ \\
\hline 1 & $\overline{B D}+61119$ & $\overline{F 8}$ & 8.98 & 0.590 & 5948 & 5939 & 132 & 3.01 & 0.77 & 28.15 & 6.90 \\
\hline 2 & RX J0156.4+1224 & G & 10.35 & 0.598 & 5956 & 5961 & 284 & 3.14 & 0.38 & 28.46 & 6.89 \\
\hline 3 & $\mathrm{BD}+30397 \mathrm{~A}$ & K8 & 10.12 & 1.205 & 4398 & 4380 & 215 & 2.23 & 0.03 & 29.95 & 6.24 \\
\hline 4 & HD 17662 & G5 & 8.46 & 0.720 & 5542 & 5622 & 23 & 1.89 & -0.56 & 32.00 & 6.76 \\
\hline 5 & $\mathrm{BD}+21418$ & G5 & 8.5 & 0.635 & 5843 & 5895 & 247 & 3.18 & 0.99 & 29.21 & 6.85 \\
\hline 6 & HD 20678 & $\mathrm{~K} 0 \mathrm{~V}$ & 7.954 & 0.728 & 5268 & 5263 & 33 & 1.49 & 1.09 & 29.04 & 6.75 \\
\hline 7 & HD 21051 & KIII & 6.05 & 1.187 & 4817 & 4826 & 287 & 2.76 & 1.18 & 30.07 & 6.25 \\
\hline 8 & HD 21845 & K2 & 8.250 & 0.800 & 5068 & 5076 & 210 & 2.88 & 0.62 & 30.08 & 6.67 \\
\hline 9 & HD 26913 & G8V & 6.92 & 0.680 & 5662 & 5648 & 85 & 2.51 & 1.35 & 30.67 & 6.80 \\
\hline 10 & HD 284503 & G8 & 10.48 & 0.872 & 5109 & 4997 & 161 & 2.61 & 0.01 & 29.79 & 6.60 \\
\hline 11 & HD 285840 & K1 & 4.76 & 0.702 & 5604 & 5598 & 178 & 3.02 & 0.06 & 30.23 & 6.78 \\
\hline 12 & HD 283716 & K0IV & 10.34 & 0.769 & 5403 & 5422 & 39 & 1.89 & -0.28 & 31.48 & 6.71 \\
\hline 13 & HD 31281 & G0 & 9.19 & 0.672 & 5647 & 5639 & 0 & 1.01 & 1.03 & 31.73 & 6.81 \\
\hline 14 & HD 32704 & G8V & 8.66 & 0.759 & 5338 & 5347 & 24 & 1.52 & 0.24 & 30.99 & 6.72 \\
\hline 15 & HD 287927 & G5 & 10.6 & 0.709 & 5498 & 5507 & 254 & 3.24 & 0.08 & 29.16 & 6.77 \\
\hline 16 & HD 245924 & K0IV & 9.7 & 0.880 & 5109 & 5108 & 247 & 2.84 & 0.05 & 30.69 & 6.59 \\
\hline 17 & HD 251108 & K2 & 9.97 & 1.332 & 4342 & 4339 & 115 & 1.68 & -1.54 & 29.93 & 6.10 \\
\hline 18 & $\mathrm{BD}+331646$ & K7 & 9.90 & 1.400 & 4236 & 4242 & 15.3 & 0.41 & -0.97 & 29.43 & 6.02 \\
\hline 19 & HD 79551 & G5 & 8.734 & 0.662 & 5642 & 5635 & 19 & 1.52 & 0.27 & 31.28 & 6.82 \\
\hline 20 & HD 81025 & G2III & 6.368 & 0.774 & 5298 & 5306 & 22 & 1.98 & 1.22 & 30.16 & 6.70 \\
\hline 21 & HD 82210 & G5III & 4.565 & 0.781 & 5249 & 5244 & 30 & 1.59 & 1.15 & 30.33 & 6.69 \\
\hline 22 & HD 95559 & $\mathrm{~K} 0 \mathrm{~V}$ & 8.83 & 0.872 & 5038 & 5043 & 68 & 1.96 & 0.24 & 30.42 & 6.60 \\
\hline 23 & $\mathrm{BD}+122343$ & G5 & 9.33 & 0.750 & 5347 & 5355 & 17 & 1.52 & -0.04 & 30.87 & 6.73 \\
\hline 24 & HD 101501 & G8V & 5.32 & 0.723 & 5453 & 5447 & 25 & 0.86 & 1.07 & 28.22 & 6.76 \\
\hline 25 & HD 111395 & G5V & 6.31 & 0.703 & 5508 & 5513 & 29 & 1.36 & 1.13 & 28.41 & 6.78 \\
\hline 26 & $\mathrm{BD}+232581$ & $\mathrm{~K} 4 \mathrm{~V}$ & 9.61 & 1.030 & 4709 & 4716 & 17 & 0.89 & 1.13 & 30.22 & 6.42 \\
\hline 27 & HD 126868 & G2IV & 4.844 & 0.693 & 5506 & 5497 & 98 & 2.48 & 1.23 & 30.32 & 6.79 \\
\hline 28 & HD 133640 & G2IV & 4.76 & 0.647 & 5682 & 5677 & 24 & 2.01 & 0.99 & 29.69 & 6.84 \\
\hline 29 & HD 141004 & G0V & 4.43 & 0.604 & 5868 & 5862 & 14 & 1.96 & 1.37 & 27.67 & 6.89 \\
\hline 30 & HD 340540 & K0 & 9.15 & 0.937 & 4897 & 4903 & 91 & 2.17 & 0.15 & 31.05 & 6.53 \\
\hline 31 & $\mathrm{BD}+483686$ & $\mathrm{~K} 1 \mathrm{~V}$ & 8.9 & 0.847 & 5092 & 5084 & 248 & 2.48 & 0.25 & 29.74 & 6.62 \\
\hline 32 & BD+66 1664 & G8 & 8.74 & 0.708 & 5489 & 5482 & 25 & 1.51 & 0.07 & 32.94 & 6.77 \\
\hline 33 & HD 232862 & G8II & 9.46 & 0.878 & 5048 & 5052 & 191 & 2.51 & 0.13 & 30.46 & 6.59 \\
\hline
\end{tabular}

Notes. $C$ : the effective temperature was determined from $B-V$ colour. $R$ : the effective temperature was determined from the line-depth ratios of VI $\lambda 6251.83$ and Fe I $\lambda 6252.527$.

error of $5 \%$ corresponds to an error of 0.04 dex in $\log g$. The estimated errors of $70 \mathrm{~K}$ in effective temperature and $0.05 \mathrm{mag}$ in apparent magnitude each lead to an uncertainty of 0.02 dex in $\log g$, while an error of $0.05 M_{\odot}$ in mass lead to an error of 0.03 dex in gravity. Adding these values in quadrature gives a final uncertainty $<0.2$ dex.

\subsection{Calculation of lithium abundance}

Lithium abundances (on a scale where $\log N(\mathrm{H})=12.00$ ) were obtained from comparison of the measured $\mathrm{Li} \mathrm{I} \lambda 670.8 \mathrm{~nm}$ EWs with the curve of growth calculations in non-LTE (NLTE) conditions (Pavlenko and Magazzú 1996, used the newest models (Kurucz 1993) with solar metallicity and gravities and effective temperatures in the range $\log g=3.0-4.5$ and $T_{\text {eff }}=3500-6000 \mathrm{~K}$ ).

The lithium abundances of 33 observed sample stars are reported in Table 1 . The main source of error in the derived $\log N(\mathrm{Li})$ values is the uncertainty in the effective temperature. Following the uncertainty analysis of Martín et al. (1994), we can estimate the mean uncertainty of $\log N(\mathrm{Li})$. From Fig. 5, we can see that the uncertainty of $\pm 250 \mathrm{~K}$ in $T_{\text {eff }}$ or 0.5 dex in $\log g$, translates into error bars in $\log N(\mathrm{Li})$ of about $0.4 \mathrm{dex}$ and $0.1 \mathrm{dex}$, respectively. The effects of microturbulence in the derived $\mathrm{Li}$ abundance are probably negligible.
Basri et al. (1991) showed that for $5200 \mathrm{~K}$ and an equivalent width of $\mathrm{EW}(\mathrm{Li})=0.40 \AA$, changing the microturbulence from 1.8 to $3.5 \mathrm{~km} \mathrm{~s}^{-1}$ only affects the derived abundances by -0.1 dex (King 1993). The uncertainty in $\log N(\mathrm{Li})$ is relate to the error in $\log g$, which is negligible based on consistent calculations. However, to be conservative, we adopted an uncertainty of $\sigma(\log g)= \pm 0.1$ dex in the relative Li abundances. The uncertainty in the EWs is $\pm 10 \%$, introducing an uncertainty $\leq \pm 0.1$ dex to the abundances. An uncertainty of $\pm 70 \mathrm{~K}$ in $T_{\text {eff }}$ results in an uncertainty of $\leq \pm 0.15 \mathrm{dex}$ in the abundances. Adding these values in quadrature gives a final uncertainty for the relative abundances of $\leq \pm 0.2$ dex for all but one star (HD 31281).

\subsection{Calculation of $X$-ray luminosity}

X-ray activity measures, $L_{X}$, were calculated for each star using the ROSAT All-Sky Bright Source Catalogue data. The X-ray flux, $f_{\mathrm{X}}$, was derived as

$f_{\mathrm{X}}=\mathrm{ECF} \cdot \operatorname{count}$ rate $\left(\mathrm{erg} \mathrm{cm}^{-2} \mathrm{~s}^{-1}\right)$

where ECF is the energy conversion factor given as

$\mathrm{ECF}=\left(5.30 \mathrm{HR}_{1}+8.31\right) \times 10^{12}\left(\mathrm{erg} \mathrm{cm}^{-2} \mathrm{cts}^{-1}\right)$

according to Schmitt et al. (1995) where

$L_{\mathrm{X}}=4 \pi d^{2} f_{\mathrm{X}}$ 


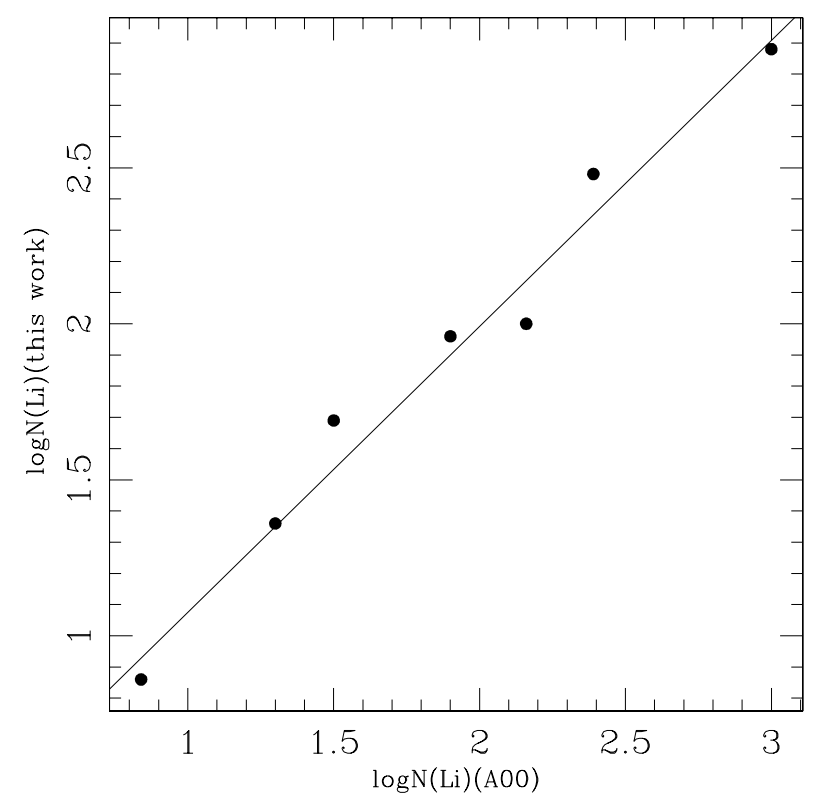

Fig. 3. A comparison of $\log N(\mathrm{Li})$ of sample stars of Christian \& Mathioudakis (2002), Wichmann et al. (2000), de Laverny et al. (2003), Takeda \& Kawanomoto (2005), Jasniewicz et al. (1999), Montes et al. (2001) with that of this work.

and $d$ is the distance to the star derived from the trigonometric parallax. The trigonometric parallax of star was taken from VizieR.

\section{Discussion and results}

For the sake of consistency, we show in Fig. 3 a comparison of $\log N(\mathrm{Li})$ derived in this study with other works (Christian \& Mathioudakis 2002; Wichmann et al. 2000; de Laverny et al. 2003; Takeda \& Kawanomoto 2005; Jasniewicz et al. 1999; Montes et al. 2001). As expected, we found only minor differences with respect to other original lithium abundances. We are confident that our lithium abundances are reliable.

Figure 4 shows the $\log N(\mathrm{Li})$ versus (vs.) the $T_{\text {eff }}$ distribution for young solar-type stars in our sample. The solid and dashed curves are the fiducial $\log N(\mathrm{Li})$ vs. $T_{\text {eff }}$ curves for the Pleiades (top envelope) and Hyades clusters, respectively (adapted from Deliyannis 2000). The figure indicates that most of our sample stars are above the Hyades track. Many stars in our sample are clearly quite young. We find that among the stars in our sample, 11 have a $\log N(\mathrm{Li})$ higher than that of the Pleiades (all of which are younger than ZAMS Pleiades stars, and should be premain sequence (PMS) stars), 15 have a $\log N(\mathrm{Li})$ between that of the Pleiades and Hyades (all are younger than Hyades stars, and should be ZAMS), and 7 have a $\log N(\mathrm{Li})$ lower than that of the Hyades (all of which should be young solar-type stars). The figure also indicates that for these young solar-type stars, no dependency of $\log N(\mathrm{Li})$ on $T_{\text {eff }}$ is discernible.

Soderblom et al. (1993a) studied lithium abundance in conjunction with rotational velocity and chromospheric activity in the Pleiades, and found that (for $0.8<B-V<1.2$ ) stars with a large EW of the Li I $6708 \AA$ line tend to have a large value of projected rotational velocity. In the $\alpha$ Per cluster, more rapid rotators also appear to have a stronger lithium abundance (Balachandran et al. 1988; Stauffer et al. 1993). These results are the same for nearby field solar-type stars (de Medeiros et al. 2000; do Nascimento et al. 2000; Cutispoto et al. 2003). On the

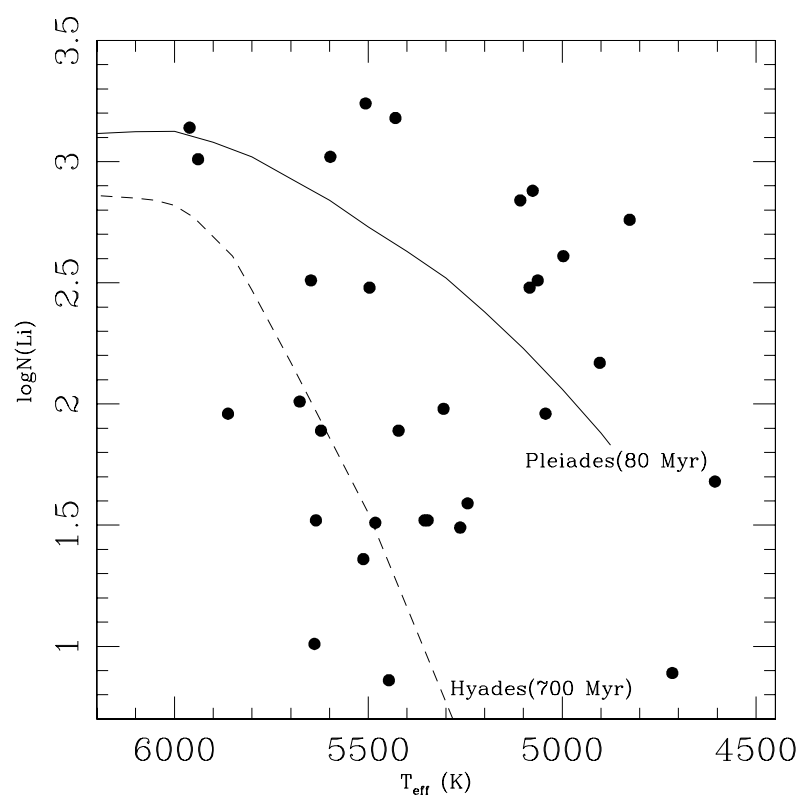

Fig. 4. Li abundance versus effective temperature for stars in the present survey. The solid and dashed curves are the fiducial $\log N(\mathrm{Li})$ vs. $T_{\text {eff }}$ curves for the Pleiades (top envelope) and Hyades, respectively (adapted from Deliyannis 2000).

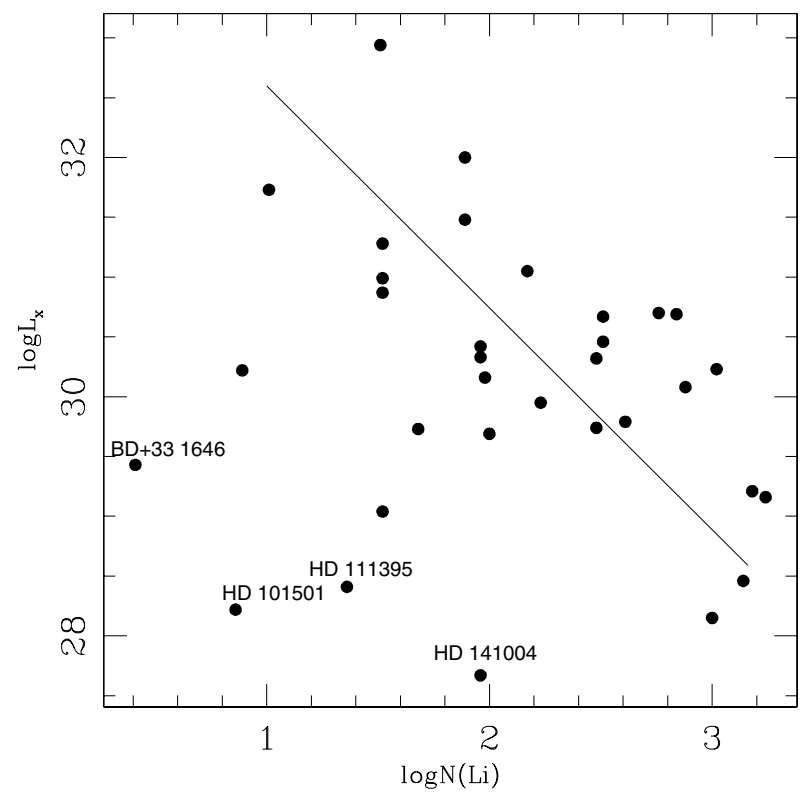

Fig. 5. Lithium abundances as a function of the X-ray luminosity $\left(\log L_{\mathrm{x}}\right)$ for the sample of young solar-type stars.

other hand, observations and data analysis show that the rapid rotation of young solar-type stars exhibits higher X-ray luminosity (Bouvier et al. 1985; Xing et al. 2007a). The mean levels of both lithium abundance and X-ray luminosity are known to decrease with decreasing projected rotational velocity (although perhaps with a large dispersion) in solar-type MS stars. Therefore, some level of correlation between lithium abundances and X-ray luminosity was expected in the nearby field young solar-type stars.

Figure 5 presents the relationship between X-ray luminosity $\left(\log L_{\mathrm{X}}\right)$ and lithium abundances for the sample of 33 (11 PMS, 15 ZAMS and 7 young MS) stars selected from the ROSAT survey. Except for four multi-star system (BD+33 1646, 


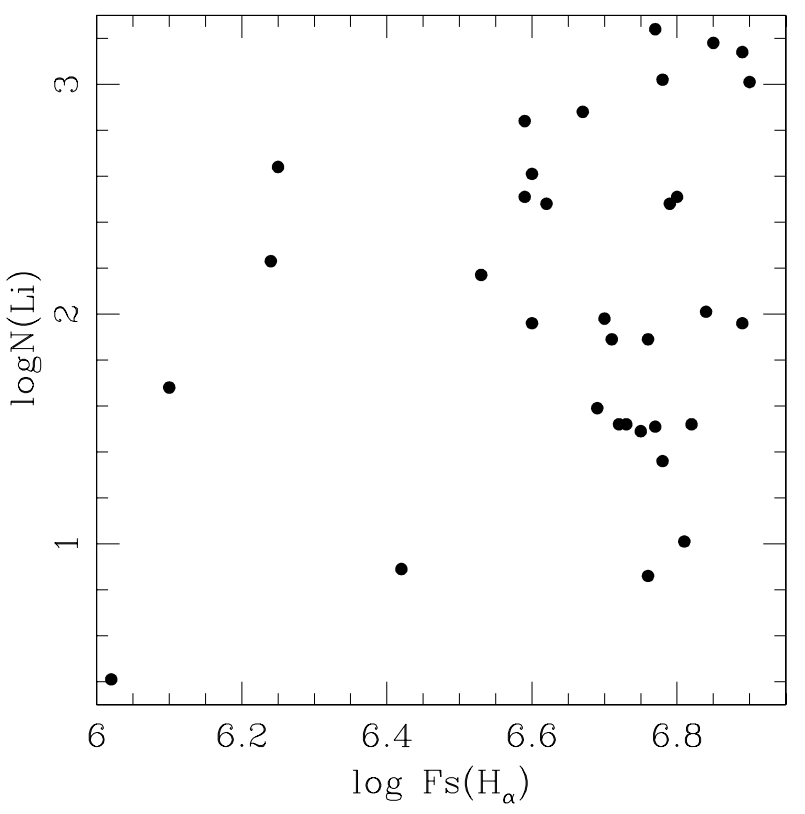

Fig. 6. Lithium abundances as a function of the $\log F_{\mathrm{s}}$ for the sample of young solar-type stars.

HD 111395, HD 101501, and HD 141004), Fig. 5 shows that there exits a clear correlation between X-ray activity and Li abundance: as the X-ray luminosity, $L_{X}$ becomes stronger, the lithium abundance decreases.

Pasquini et al. (1994) found a clear tendency for nearby chromospherically active solar-type stars to have high $\log N(\mathrm{Li})$. Similar results were found in X-ray samples or EUV-selected active stars (Favata et al. 1993; Tagliaferri et al. 1994, 2000; Jeffries 1995). Stellar activity can then be considered a good indicator of age for solar-type stars (e.g., Soderblom et al.1991; Pizzolato et al. 2000). We therefore searched for a correlation between Li abundance and chromospheric activity in our sample stars. We determined the equivalent widths of $\mathrm{H}_{\alpha}$ line $\left(\mathrm{EW}\left(\mathrm{H}_{\alpha}\right)\right)$ and converted $\mathrm{EW}\left(\mathrm{H}_{\alpha}\right)$ to absolute chromospheric flux $\left(\log F_{\mathrm{S}}\right)$ at the stellar surface by using the calibration of Hall (1996) as a function of $(B-V)$. In Table 1, we present/provide the $\mathrm{EW}\left(\mathrm{H}_{\alpha}\right)$ and $\log F_{\mathrm{S}}$ of these stars. In Fig. 6, we present the $\log F_{\mathrm{S}}$ versus the lithium abundances. However, Fig. 6 shows that the data of these 33 stars (consisting of PMS, single MS stars, RS CVn, BY Dra, and other binary systems) are highly scattered, perhaps since the close binary systems have enhanced activities (Fernández-Figueroa et al. 1993) or others.

When considering the main features of the rotation evolution of low mass, we find that late-type stars exhibit a strong PMS spin up (in the phase from moderate rotation in the T Tauri to ultrafast rotation at ZAMS) and an increase in rotational period (spin down) with increasing age for main-sequence stars (e.g., Bouvier 1994; Soderblom et al. 1993b; Collier Cameron et al. 1995; Keppens et al. 1995). Therefore, the EW(Li) decreases with decreasing $P_{\text {rot }}$ when a star evolves in the PMS phase, whereas $\mathrm{EW}(\mathrm{Li})$ decreases with increasing $P_{\text {rot }}$ (decreasing rotational velocity) when stars evolve in the MS phase (Xing et al. 2007b). However, as the rapid rotation of young solar-type stars increases shows their higher X-ray luminosity similarly increases (Bouvier et al. 1985; Xing et al. 2007a). It is easy to understand that when solar-type stars evolve in the PMS phase, their lithium abundance decreases as the X-ray luminosity $\left(L_{X}\right)$ becomes stronger. In contrast, in the MS phase, more rapid rotators appear to have a stronger X-ray flux $\left(L_{\mathrm{X}}\right)$ and higher lithium abundances. The results of the present study are in good agreement with the results of Pasquini et al. (1994), Favata et al. (1993,1995), Tagliaferri et al. (1994, 2000), and Jeffries (1995).

Lithium and X-ray activity appear to be correlated for the 33 young solar-type stars in our sample. These results are in good agreement with the rotation evolution model (e.g., Bouvier 1994; Soderblom et al. 1993b; Collier Cameron et al. 1995; Keppens et al. 1995), and Li could serve as a "clock" of stars that develops in the PMS phase (Drake et al. 2003). This correlation for our present sample appears to be simply an age effect, with lithium decaying and activity increasing with age for PMS solar-type stars. Our results, which show agreement with recent findings from X-ray surveys, confirm the presence of very active young stars close to the Sun.

\section{Conclusions}

In summary, we have presented high-resolution spectroscopic observations for a sample of 33 young solar-type stars discovered in the X-ray wavelength range during an ROSAT all-sky survey. On the basis of our observations, we have derived the lithium abundances of the stars by comparing the measured Li I $\lambda 670.8 \mathrm{~nm}$ EWs with the curve of growth calculations in nonLTE (NLTE) conditions (Pavlenko \& Magazzú 1996). We have found a clear correlation between X-ray activity and lithium abundances: as the X-ray luminosity, $L_{\mathrm{x}}$, becomes stronger, the lithium abundance decreases in our sample stars. The results of the current study provide additional evidence that this correlation exists for a large number of solar-type stars. A correlation appears to exist between lithium abundances and the age of stars. In addition, we have confirmed that there are very active young stars close to the Sun.

Acknowledgements. We would like to thank Prof. Gang Zhao for helpfull discussions. The authors gratefully acknowledge the many and detailed positive contributions made by the referee. This work was supported by the jointing fund of the Astronomy of the National Natural Science Foundation of China and the Chinese Academy of Sciences, under Grants No. 10878015 and partially Supported by the Open Project Program of the Key Laboratory of Optical Astronomy, National Astronomical Observatories, Chinese Academy of Sciences.

\section{References}

Balachandran, S., Lambert, D. L., \& Stauffer, J. R. 1988, ApJ, 333, 267

Barrado y Navascués, D., \& Stauffer, J .R. 1996, A\&A, 310, 879

Basri, G., Martín, E. L., \& Bertout, C. 1991, A\&A, 252, 625

Boesgaard, A. M. 1991, ApJ, 370, L95

Boesgaard, A. M., \& Tripicco, M. J. 1986, ApJ, 303, 724

Boesgaard, A. M., Deliyannis, C. P., Stephens, A., et al. 1998, ApJ, 493, 206

Bouvier, J. 1994, ASPC, 64, 151

Bouvier, J., \& Bertout, C. 1985, Msngr, 39, 33

Bouvier,J., Bertout,C., Benz, W., et al. 1985, in Nearby Molcular Clouds, ed. G. Serra, Lect. Notes Phys., 222

Casagrande, L., Portinari, L., \& Flynn, C. 2006, MNRAS, 373, 13

Cayrel, R., Cayrel de Stroebel, G., Campbell, B., et al. 1984, ApJ, 283, 205

Chen, Y. Q., Nissen, P. E., Zhao, G., et al. 2000, A\&AS, 141, 491

Christian, D. J., \& Mathioudakis, M. 2002, AJ, 123, 2796

Collier Cameron, A., Campbell, C. G., \& Quaintrell, H. 1995, A\&A, 298, 133

Cutispoto, G., Tagliaferri, G., de Medeiros, J. R., et al. 2003, A\&A, 397, 987

de Laverny, P., do Nascimento, J. D. Jr., \& Lébre, A. 2003, A\&A, 410, 937

de Medeiros, J. R., do Nascimento, J. D., Sankarankutty, S., et al. 2000, A\&A, 363,239

Deliyannis, C. P. 2000, ASPC, 198, 235

do Nascimento, J. D., Charbonnel, C., Lebre, A., et al. 2000, A\&A, 357, 931

Drake, N. A., de La Reza, R., da Silva, L., et al. 2003, BASBr, 23, 107

Duncan, D. K. 1981, ApJ, 248, 651

Duncan, D. K., \& Jones, B. F. 1983, AJ, 271, 663

Favata, F., Barbera, M., Micela, G., et al. 1993, A\&A, 277, 428

Favata, F., Barbera, M., Micela, G., et al. 1995, A\&A, 295, 147 
Fernández-Figueroa, M. J., Barroado, D., De Castro, E., et al. 1993, A\&A, 274, 373

Gray, D. F., \& Johanson, H. L. 1991, PASP, 103, 439

Hall, J. C. 1996, PASP, 108, 313

Gregorio-Hetem, J., \& Hetem, A. 2002, MNRAS, 336, 197

Hobbs, L. M., \& Pilachowski, C. 1986, ApJ, 309, L17

Jasniewicz, G., Parthasarathy, M., \& de Laverny, P. 1999, A\&A, 342, 831

Jeffries, R. D. 1995, MNRAS, 273, 559

Jones, B. F., Fischer, D., Shetrone, M., et al. 1997, AJ, 114, 352

Keppens, R., MacGregor, K. B., \& Charbonneau, P. 1995, A\&A, 294, 469

King, J. R. 1993, AJ, 105, 1087

Kurucz, R .L. 1993, ATLAS9 Stellar Atmosphere Program and $2 \mathrm{~km} \mathrm{~s}^{-1}$ grid (Kurucz CD-ROM No. 13)

Martín, E. L., Rebolo, R., Magazzú, A., et al. 1994, A\&A, 282, 503

Montes, D., López-Santiago, J., \& Fernández-Figueroa, M. J. 2001, A\&A, 379, 976

Nissen, P. E., Hog, E., \& Schuster, W. J. 1997, ESASP-402, Hipparcos Venice97, ed. B. Barrrick, 225

Palla, F., Randich, S., Flaccomio, E., \& Pallavicini, R. 2005, ApJ, 626, L49

Pasquini, L., Liu, Q., \& Pallavicini, R. 1994, A\&A, 287, 191

Pavlenko, Ya. V., \& Magazzú, A. 1996, A\&A, 311, 961

Pizzolato, N., Maggio, A., \& Sciortino, S. 2000, A\&A, 361, 614
Preibisch, T. 1997, A\&A, 320, 525

Randich, S., Pallavicini, R., Meola, G., et al. 2001, A\&A, 372, 862

Rebolo, R. 1989, Astrophys. Space Sci., 157, 47

Rebolo, R., Molaro, P., \& Beckman, J. 1988, A\&A, 192, 192

Rebolo, R., \& Beckman, J. E. 1988, A\&A, 201, 267

Schmitt, J. H. M. M., Fleming, T .A., \& Giampapa, M. S. 1995, ApJ, 450, 392

Soderblom, D. R., Duncan, D. K., \& Johnson, D. R. H. 1991, ApJ, 355, 722

Soderblom, D. R., Jones, B. F., Balachandran, S., et al. 1993a, AJ, 106, 1059

Soderblom, D. R., Stauffer, J. R., MacGregor, K. B., et al. 1993b, ApJ, 409, 624

Spite, F., \& Spite, M. 1982, A\&A, 115, 357

Stauffer, J. R., Prosser, C. R., Giampapa, M. S., et al. 1993, AJ, 106, 229

Tagliaferri, G., Cutispoto, G., Pallavicini, R., et al. 1994, A\&A, 285, 272

Tagliaferri, G., Pastori, L., Cutispoto, G., et al. 2000, in The Light Elements and their Evolution, ed. L. da Silva, R. de Medeiros, \& M. Spite, IAU Symp., 198, 366

Takeda, Y., \& Kawanomoto, S. 2005, PASJ, 57, 45

Tschäpe, R., \& Rüdiger, G. 2001, A\&A, 377, 84

Wichmann, R., Torres, G., \& Melo, C. H. F. 2000, A\&A, 359, 181

Xing, L. F. 2010, ApJ, 723, 1542

Xing, L. F., Zhang, X. B., \& Wei, J. Y. 2007a, New Astron., 12, 364

Xing, L. F., Shi, J. R., \& Wei, J. Y. 2007b, New Astron., 12, 265

Zhao, G., \& Li, H. B. 2001, Chin. J. Astron. Astrophys., 1, 555 